\title{
Diagnóstico da poluição ambiental em área de preservação no município de Lagoa Seca - Paraíba
}

\section{Diagnosis of environmental pollution in conservation area in the municipality of Lagoa Seca-Paraíba}

\author{
Júlia Soares Pereira ${ }^{1}$, Jean Pereira Guimarães ${ }^{2}$, Maria Sallydelândia Sobral Farias ${ }^{3}$
}

Resumo: A água é um recurso natural renovável finito, no entanto sua qualidade vem se agravando decorrente da crescente população e à falta de políticas públicas para a preservação deste recurso. Nesse sentido, este trabalho teve por objetivo diagnosticar a Área de Preservação Ambiental (APA) do Posto de Fomento, localizado no Centro de Ciências Agrárias e Ambientais - CCAA, da Universidade Estadual da Paraíba Campus II, onde funciona também a Escola Agrícola Assis Chateaubriand, caracterizado pelos experimentos práticos realizados pelos estudantes técnicos e graduandos, que fica em torno de duas comunidades rurais, Vila Florestal e Ipuarana no município de Lagoa Seca - Paraíba. Foram avaliados os problemas de degradação da área (resíduos sólidos, desmatamento, invasão e retirada de riquezas dentro da área) e qualidade da água de uma fonte dentro da reserva. Assim, constatou-se que em muitos pontos da área há elevado grau de degradação, através da exploração dos recursos naturais e lançamento de resíduos sólidos. A água analisada se enquadra na classe $\mathrm{C}_{2}$, água de salinidade média (CE entre 250 e $750 \mu \mathrm{S} . \mathrm{Cm}^{-1}$ a $25^{\circ} \mathrm{C}$ ), podendo ser usada sempre que houver um grau de lixiviação, mostrando boa qualidade para irrigação das culturas existentes. Plantas com moderada tolerância aos sais podem ser cultivadas, na maioria dos casos, sem necessidade de práticas especiais de controle de salinidade.

Palavras-chave: degradação, recuperação, recursos hídricos.

\begin{abstract}
Water is a finite natural renewable resource, however its quality deteriorated due to population growth and the public policies for the preservation of this resource. Thus, this study aimed to diagnose the Environmental Preservation Area (APA) of the Posto de Fomento, located in the center of agrarian and environmental sciences State University of Paraíba, which also operates the School Agricultural Assis Chateaubriand, characterized by practical experiences performed by technicians and graduate students, and is located around two communities, Vila Florestal and Ipuarana. Degradation problems of the area and quality of a water source inside the reserve were evaluated. Thus, it was found that in many parts of the field there is a high degree of degradation, through the exploitation of natural resources and release of waste. The water analyzed falls within the class $\mathrm{C} 2$ medium salinity water $\left(250\right.$ to $750 \mu \mathrm{S} \mathrm{Cm}^{-1}$ at $\left.25^{\circ} \mathrm{C}\right)$ and can be used whenever there is a degree of leaching, showing good quality for irrigation of cultures. Plants with moderate tolerance to salts can be cultivated in most cases without the need for special measures to control salinity.
\end{abstract}

Key words: degradation, recovery, water resources.

\footnotetext{
*Autor para correspondência

Recebido para publicação em 21/07/2014; aprovado em 06/02/2015

${ }^{1}$ Eng. Agrícola, Doutoranda em Engenharia Agrícola UAEA/CTRN/UFCG, Campina Grande, PB, Rua Aprígio Veloso, n 882, Bairro Universitário, CEP 58.429.900. E-mail: julia_eng@hotmail.com

${ }^{2}$ Eng. Agrícola, Mestrando em Engenharia Agrícola UAEA/CTRN/UFCG, Campina Grande, PB, Rua Aprígio Veloso, n 882, Bairro Universitário, CEP: 58.429.900. E-mail: jean.p.guimaraes@ gmail.com

${ }^{3}$ Eng. Agrícola, Dr ${ }^{\mathrm{a}}$. Engenharia Agrícola, Professora UAEA/CTRN/UFCG, Campina Grande, PB, Rua Aprígio Veloso, n 882, Bairro Uni versitário, CEP: 58.429.900. E-mail: sally_farias@yahoo.com.br
} 


\section{INTRODUÇÃO}

Segundo Araújo e Xavier (2010), as questões relacionadas com agressão ao meio ambiente, concentram ao mesmo tempo, problemas de cunho ético, político, econômico, social e cultural. A sociedade necessita modificar a forma de pensar a relação do ser humano com a natureza entre si, propondo e questionando formas de desenvolvimento que estejam efetivamente voltadas para melhoria da qualidade de vida dos seres humanos, ao mesmo tempo comprometida com a preservação da natureza e com o respeito ao meio ambiente.

Segundo Lima et al. (2009), a disponibilidade de água é a primeira prioridade para ser humano, seguida do acesso ao suprimento de alimentos, o que faz com que a agricultura seja um componente dominante da economia mundial, tanto pela necessidade da água para viabilizar a produção agrícola como o atendimento da demanda humana por alimentos.

Portanto, a agricultura exige uma interação dos elementos da terra e da água, em que medidas devem ser adotadas para assegurar que as atividades inerentes às suas demandas não afetem adversamente a qualidade da água, de modo que seus usos subsequentes, para diferentes propósitos, não sejam prejudiciais ao ambiente e à saúde humana (LIMA, 2010).

Esta pesquisa foi realizada na Área de Preservação Ambiental do Posto de Fomento, localizado no Centro de Ciências Agrárias e Ambientais - CCAA, da Universidade Estadual da Paraíba Campus II, onde funciona também a Escola Agrícola Assis Chateaubriand, caracterizado pelos experimentos práticos realizados pelos estudantes técnicos e graduandos de Agroecologia. A reserva foi cedida pelo governo federal para estudos, análises e recuperação da área degradada dentro da reserva, que fica em torno de duas comunidades, Vila Florestal e Ipuarana, no município de Lagoa Seca - Paraíba. Este trabalho teve como objetivo diagnosticar a degradação ambiental da reserva, como desmatamento, perda de solo, análise de resíduos sólidos, entre outros dados pontuais da área, e analisar a qualidade de água em fontes dentro da mesma e no açude próximo à comunidade, para posterior utilização na irrigação de produção de mudas.

\section{MATERIAL E MÉTODOS}

O espaço geográfico de estudo foi a Área de Preservação Ambiental, Posto de Fomento - Produção de Mudas situada no Sítio Imbaúba, zona rural do município de Lagoa Seca $\mathrm{PB}$, com latitude $07^{\circ} 10^{\prime} 15^{\prime \prime}$, longitude $35^{\circ} 51^{\prime} 13^{\prime \prime}$ e altitude de 634 metros, perfazendo um total de $68,3 \mathrm{~km}^{2}$. Seu clima é o tropical úmido, com temperatura média anual em torno de $22{ }^{\circ} \mathrm{C}$, sendo a mínima de $18^{\circ} \mathrm{C}$ e a máxima de $33^{\circ} \mathrm{C}$.

Para análise da área foram realizadas visitas semanais para obtenção de dados, utilizando-se de máquina fotográfica para registro de locais pontuais, análise da água com material esterilizado, devidamente adequado, instrumentos de pesquisa e entrevista com a comunidade local. Observou-se que a área ambiental esta marcada pelo depósito inadequado de resíduos sólidos, processo de erosão laminar, pois a intensificação do uso do solo e a remoção da cobertura vegetal é visível nesta área, apresentando solo exposto às intempéries facilitando a perda do mesmo, sobretudo após precipitações. Também foi percebido que as cercas de madeiras encontram-se bem danificadas o que facilita a entrada de animais e pessoas na área, contribuindo ainda mais para o processo de degradação (Figura 1A; 1B). Em processos de recuperação de áreas degradadas o isolamento da área é de fundamental importância para minimizar os impactos causados principalmente pela ação humana das comunidades rurais vizinhas.

A população que reside próximo à área de preservação lança seus resíduos sólidos diretamente na área, mesmo existindo coleta de resíduos sistemática, por parte do órgão público local, mostrando a importância de se executar trabalho de educação ambiental nas comunidades localizadas próximas à área. Observou-se também in loco que a parte da área que é cercada apenas com madeira e arame apresenta maior vulnerabilidade de invasão.

Figuras 1. Resíduos sólidos em vários pontos do entorno da reserva (A), crianças em contato com os resíduos e demonstração de cercas de estaca de sabiá e arame, além de solo exposto, voçorocas e desnível em toda a área (B).

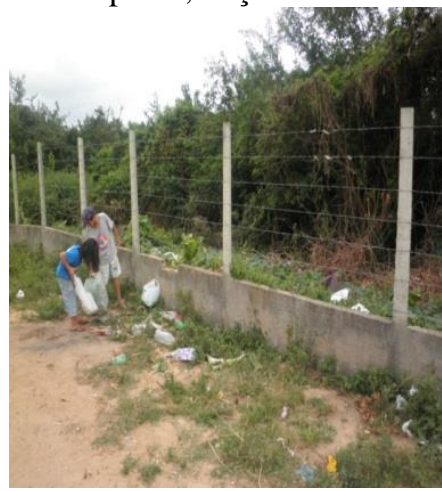

(A)

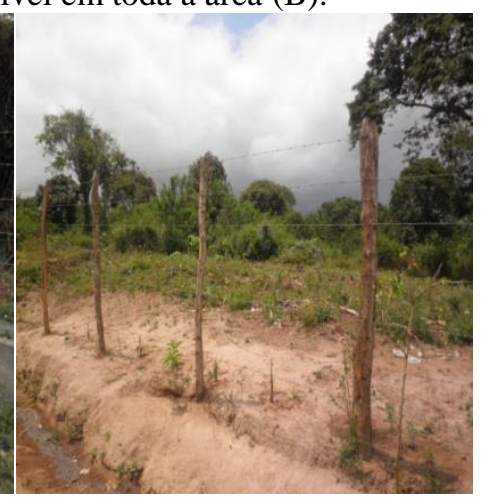

(B)
Foi realizada análise da água para fins de irrigação, tanto em um dos açudes próximo a comunidade, quanto dentro da própria reserva ambiental (Figura 2A; 2B).

Figura 2. Reservatórios. Açude próximo à comunidade de Vila Florestal e Ipuarana (A), e Depósito de água dentro da área, advindo de nascente (B).

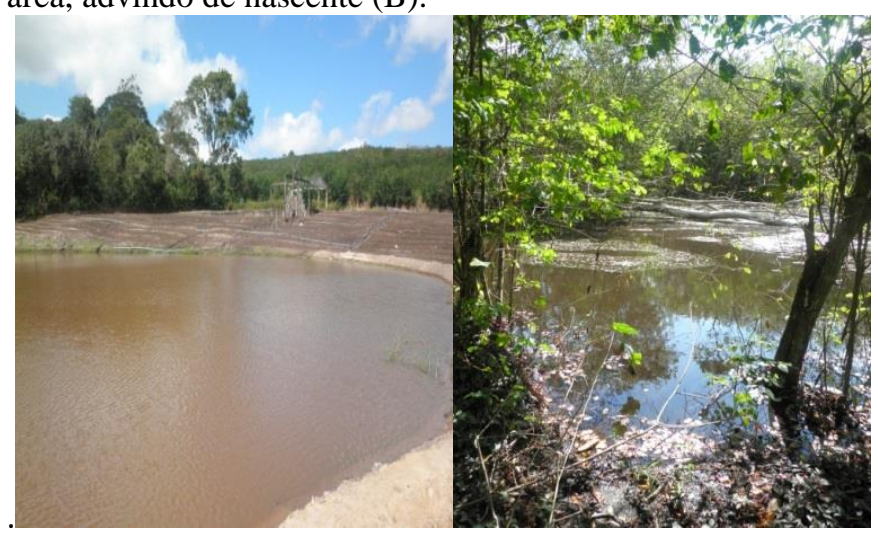

(A)

(B)

\section{RESULTADOS E DISCUSSÃO}

O desmatamento da vegetação nativa é observado em vários pontos da área. Sendo agredida constantemente através da retirada de madeira pelos integrantes das comunidades da Vila Florestal e Ipuarana para utilização em necessidades domesticas diversas. O despejo de resíduos sólidos e líquidos 
são constantes na área. Além de ser considerada uma área de Preservação Permanente devido seus aos atributos ambientais em diversos pontos a área apresenta inclinação de $45^{\circ}$, caracteriza-se, também, como Área de Preservação Permanente. Segundo o Código Florestal (art. 10) determina que nas áreas com inclinação entre 25 e $45^{\circ}$ é vedada a derrubada de florestas, permitida apenas a exploração seletiva em regime de manejo sustentável

De acordo com Empresa Brasileira de Pesquisa Agropecuária (EMBRAPA) e com o Conselho Nacional de Desenvolvimento Científico e Tecnológico - CNPq, o desmatamento é caracterizado pela prática de corte, capina ou queimada (por fogo ou produtos químicos), que leva à retirada da cobertura vegetal existente em determinada área, para fins de pecuária, agricultura ou expansão urbana. A devastação florestal preocupa brasileiros e ambientalistas do mundo todo, pois interfere na fauna, destrói espécies da flora, contribui para a poluição da água, do ar, das chuvas ácidas, do efeito estufa e a comercialização ilegal de madeiras nobres (LEÃO, 2000).

As áreas de proteção ambiental pertencem ao Sistema Nacional de Unidades de Conservação, regulado pela Lei
9.985 de 18 de julho de 2000. É uma área em geral extensa, com certo grau de ocupação humana, dotada de atributos abióticos, bióticos, estéticos ou culturais, especialmente importantes para a qualidade de vida e o bem-estar das populações humanas, e tem como objetivos básicos proteger a diversidade biológica, disciplinar o processo de ocupação e assegurar a sustentabilidade do uso dos recursos naturais, além de serem fonte de sementes e apresentarem flora e fauna típica e única em cada região existente.

Assim para melhor assegurar a conservação e desenvolvimento de pesquisas é preciso inserir nas escolas locais a importância dessas "unidades de estudo", além de grande mobilização nas comunidades em relação a educação ambiental.

Nas visitas realizadas em campo observou-se visualmente, que o açude próximo à área de preservação apresenta-se excesso de sedimentos devido a falta de vegetação na mata ciliar, pequenos reservatórios dentro da área em processo de eutrofização, comprometendo a demanda hídrica para possíveis sistemas de irrigação e desenvolvimento de viveiro de mudas. Na Tabela 1 apresentase os parâmetros físicos e químicos de água.

Tabela 1 - Parâmetros físicos e químicos da água do açude e da nascente.

\begin{tabular}{ccc}
\hline & Análise da Água & \\
\hline & & Valor para fonte da reserva \\
\hline Parâmetros observados & Valor para o açude & 6,00 \\
\hline $\mathrm{pH}$ & 6,29 & 262 \\
Condutividade $\left(\mu \mathrm{S} . \mathrm{Cm}^{-1}\right)$ & 482 & 0,52 \\
Cálcio $(\mathrm{mg} / \mathrm{L})$ & 0,87 & 0,28 \\
Magnésio $(\mathrm{mg} / \mathrm{L})$ & 1,34 & 1,64 \\
Sódio $(\mathrm{mg} / \mathrm{L})$ & 2,56 & 0,18 \\
Potássio $(\mathrm{mg} / \mathrm{L})$ & 0,38 & 0,74 \\
Bicarbonatos $(\mathrm{mg} / \mathrm{L})$ & 1,86 & 1,92 \\
Cloretos $(\mathrm{mg} / \mathrm{L})$ & 2,9 & 2,60 \\
Classe de água & 2,44 & $\mathrm{C} 2$
\end{tabular}

Em água destinada à irrigação de culturas anuais ou perenes a faixa de $\mathrm{pH}$ adequada varia de 6,5 a 8,4. Valores fora desta faixa podem provocar deterioração de equipamentos de irrigação. Nos reservatórios analisados os valores estão dentro do limite permitido para o destino de irrigação.

A água analisada se enquadra na classe $\mathrm{C}_{2}$, ou seja água de salinidade média (CE entre 250 e $750 \mu \mathrm{S} . \mathrm{Cm}^{-1}$ a $25^{\circ} \mathrm{C}$ ). Podendo ser usada sempre que houver um grau de lixiviação. Plantas com moderada tolerância aos sais podem ser cultivadas, na maioria dos casos, sem necessidade de práticas especiais de controle de salinidade.

Quanto a sodicidade para valores menores que três não existe nenhuma restrição de uso. As concentrações médias de magnésio estiveram entre o valor mínimo de $0,28 \mathrm{mg} / \mathrm{l}$, no açude, e o valor máximo de $1,34 \mathrm{mg} / \mathrm{l}$, na fonte próxima a reserva. Estes valores estão bem abaixo do limite recomendado para águas de irrigação, que varia de 0 a 60 $\mathrm{mg} / \mathrm{l}$. Quanto ao potássio variações de 0 a $78 \mathrm{mg} / \mathrm{l}$ são normais em águas de irrigação, valores não ultrapassados na pesquisa (AYRES; WESTCOT, 1991).

\section{CONCLUSÕES}

Um trabalho de educação ambiental nas comunidades circunvizinhas da área de reserva é de fundamental importância para implantação de planejamento ambiental da área, incorporando ações de planejamento estratégico, que venham a colaborar com a gestão sustentável do ambiente em análise, pois não existe projeto de recuperação ambiental que funcione bem, sem o envolvimento total da comunidade.

$\mathrm{O}$ isolamento da área deve ser realizado para aumentar a dificuldade de penetração de pessoas e animais na área, que contribuem para aumento da degradação da vegetação nativa. As áreas apontadas como de erosão nas margens da área da reserva devem ser recuperadas com objetivo de evitar perda de solo, minimizar o aumento de deposito de sedimentos no açude e controlar o processo erosivo.

Nas fontes de água degradadas dentro da reserva pelo excesso de vegetação no seu leito, deve ser realizada a limpeza e aprofundamento destes mananciais, fundamentais para a manutenção dos ecossistemas locais. Além disso, o aproveitamento das fontes de água para irrigação de mudas e no controle de incêndios na própria reserva se faz relevante.

As áreas de Preservação Ambiental citada no artigo 14, inciso da lei do SNUC (Sistema Nacional de Unidades de 
Conservação, lei n. ${ }^{\circ} 9.985$ de 18/07/00), a APA (Área de Proteção Ambiental) faz parte do grupo das unidades de conservação de uso sustentável e são de responsabilidade dos órgãos ambientais como potenciais gestores e fiscalizadores, não entanto, esta fiscalização é bastante falha.

\section{REFERÊNCIAS BIBLIOGRÁFICAS}

ARAÚJO, M. S.; XAVIER, L. A. Identificação da proposta do projeto de Recuperação da Área Degradada do Posto de Fomento - Produção de Mudas da Universidade Estadual da Paraíba Campus II Lagoa Seca. 2010.
AYRES, R. S.; WESTCOT, D. W. A qualidade da água na agricultura. Water Quality for Agriculture. Tradução H.R. Ghey e J. F. de Medeiros, p. 217, 1991.

AYRES, R. S.; WESTCOT, D. W. A qualidade da água na agricultura. Estudos, irrigação e drenagem. p. 153, 1999.

LEÃO, R. M. A floresta e o homem. Editora da Universidade de São Paulo. Instituto de Pesquisas e Estudos Florestais - IPEF. São Paulo, Brasil, 2000.

LIMA, V. L. A. Curso de Capacitação em Hidrometria das Micro Bacias Hidrográficas do Semiárido Brasileiro. Módulo de Qualidade da Água. p. 57, 2010. 